\title{
Comportement mécanique de géocellules \\ sous impact. Application aux ouvrages pare-blocs
}

S. LAMBERT ${ }^{1,2}$

stephane.lambert@ grenoble.cemagref.fr

Ph. GOTTELAND²

philippe.gotteland@ ujf-grenoble.fr

\section{BERTRAND 1}

david.bertrand@ grenoble.cemagref.fr

\section{F. NICOT ${ }^{1}$}

francois.nicot@ grenoble.cemagref.fr

1 Cemagref ETNA BP 76 38402 Saint-Martin-d'Hères

2 Laboratoire 3S-R, UMR

Domaine universitaire BP 53

38041 Grenoble Cedex 9

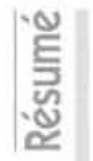

\begin{abstract}
Le comportement sous impact d'éléments géocomposites cellulaires constitutifs d'ouvrages de protection contre les chutes de blocs est étudié expérimentalement. La réponse de cellules sollicitées par largage vertical d'un impactant sous des énergies allant jusqu'à $18 \mathrm{~kJ}$ est analysée. L'influence du matériau de remplissage, des conditions aux limites de la cellule et de la hauteur de chute de l'impactant est discutée. La capacité d'atténuation des efforts transmis par la cellule lorsqu'elle est impactée dépend du couple (matériau de remplissage, conditions aux limites). Ces résultats sont ensuite comparés avec ceux issus de simulations numériques menées par la méthode aux éléments distincts.
\end{abstract}

Mots-clés : chute de blocs, protection, merlon, impact, cellule, gabion, géomatériaux, dissipation, expérimental, numérique.

\section{Mechanical behaviour of geocells subjected to impact. Application to rock fall protection structures}

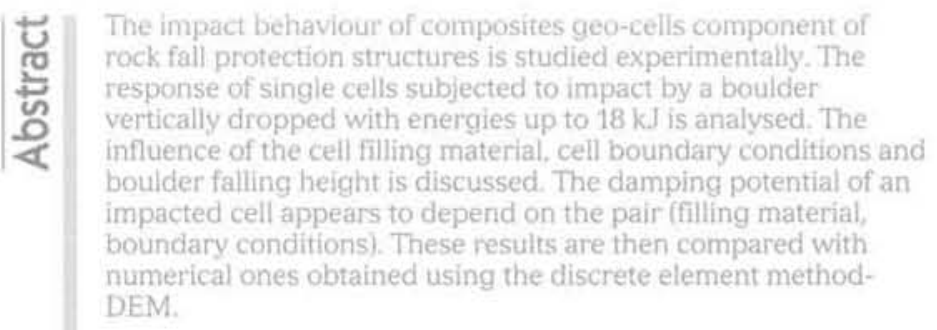

Key words : rock fall, protection, dyke, impact, cell, gabion, geo-

materials, dissipation, experiments, numerical. 


\section{Introduction}

Les chutes de blocs rocheux, toutes dimensions confondues, sont les aléas naturels les plus fréquents en zones montagneuses. Chaque année, et principalement en hiver, cet aléa cause interruptions de circulation, destructions d'infrastructures, voire atteintes mortelles aux personnes, même lors d'événements de faible énergie. Les parades contre cet aléa peuvent être actives, par une action au niveau de la zone de départ, ou passives, par une action sur le phénomène une fois développé. Dans le premier cas il s'agit d'empêcher le départ des blocs, par exemple avec des filets plaqués sur la falaise. Dans le second cas, qui nous intéresse ici, l'objectif est d'agir sur la trajectoire du bloc, le plus souvent en visant son immobilisation. Différents types de structures peuvent ètre mises en cuvre : écran de filets, casquettes, merlons (Descceudres, 1997) dont le choix sera guidé par l'énergie du bloc attendu à l'emplacement prévu pour l'ouvrage, mais également par la nature et la distance de l'enjeu à protéger. La hauteur de passage des blocs, déterminée par l'étude trajectographique, est également un paramètre dimensionnant essentiel. Les merlons sont des ouvrages construits en élévation par rapport au terrain naturel, associés à une fosse de réception, et dont l'aptitude à dissiper l'énergie cinétique du bloc incident tient à leur masse importante. La technique la plus courante en France consiste à renforcer par des inclusions souples de type géosynthétique le corps du remblai afin d'assurer sa stabilité statique et de réaliser le parement à l'amont, exposé à l'impact, à l'aide de pneus juxtaposés et superposés (procédé Pneutex). Le renforcement permet notamment le raidissement du parement à l'amont qui limite le risque de franchissement. Le parement à l'aval ne doit pas se déformer et doit généralement satisfaire des conditions d'intégration paysagère.

Cependant, le dimensionnement des merlons demeure empirique et ne tient pas réellement cormpte de la réponse à la sollicitation dynamique. Le critère dimensionnant est la stabilité propre, en statique, de l'ouvrage aux caractéristiques dimensionnelles imposées par l'enveloppe géométrique.

Un développement innovant pour la conception des merlons est la réalisation d'ouvrages sandwichs par utilisation de géocellules (Fig. 1). Les géocellules sont des éléments préfabriqués de forme géométrique régulière et remplis de géomatériaux, tels que les gabions. Elles confèrent à la structure une modularité spatiale et autorisent la mise en ceuvre simplifiée des differentes couches du sandwich. Dans l'étude présentée, les géocellules élémentaires sont constituées d'une cage grillagée métallique remplie de géomatériaux du site (gravier, blocs, tout-venant...) ou de matériaux aux caractéristiques spécifiques (matériaux anthropiques tels que des pneumatiques transformés...). Les caractéristiques mécaniques des cellules peuvent ainsi être adaptées aux différentes fonctions à remplir au sein de l'ouvrage. Les espacements et déplacements entre cellules sont rendus possibles ou non en fonction de leur position. Une telle conception vise à concentrer sur le parement à l'amont les déformations et les dégradations résultant de l'impact lors de l'interception d'un bloc rocheux. L'énergie du bloc est dissipée au niveau des cellules de parement et de noyau: la partie de la structure à l'aval est alors peu ou pas sollicitée et peut ainsi être réduite en dimensions.
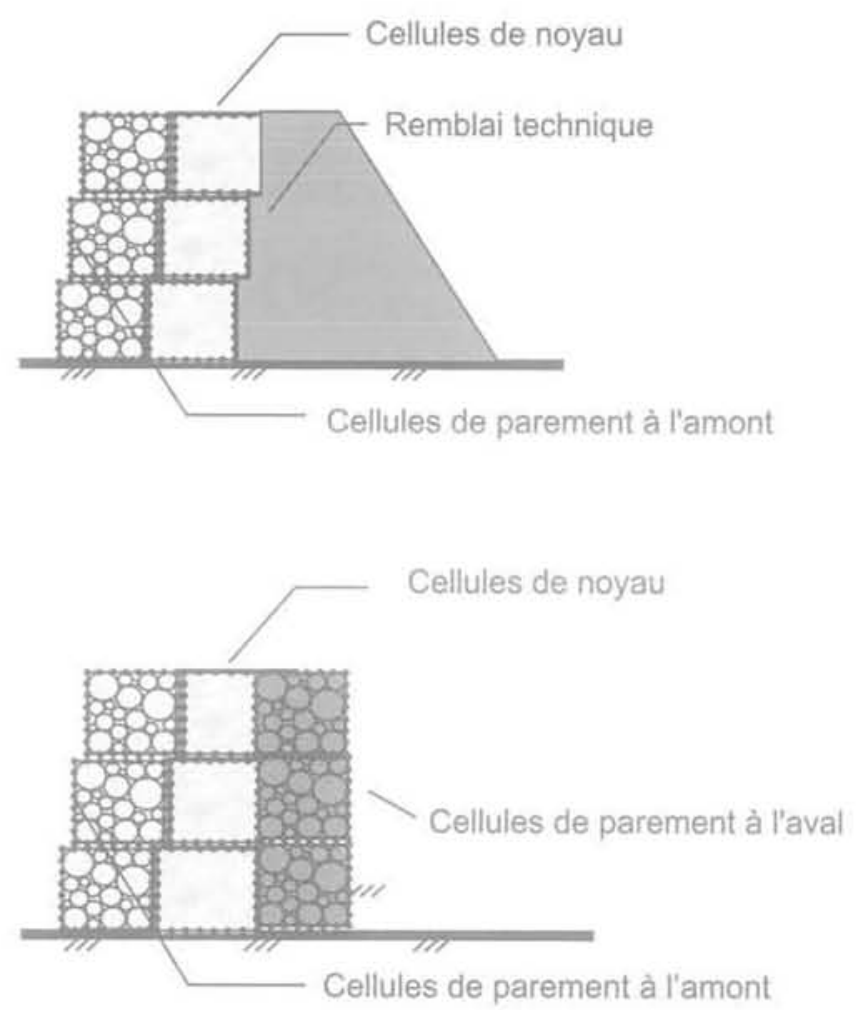

คG. 1 Principe des ouvrages par assemblages de cellules.

Principle of cellular structures.

Un autre intérêt de ce type de structure réside dans la facilité d'entretien présagée au cours de la vie de l'ouvrage et en particulier la réparation en cas de dégradations avérées suite à un impact par un bloc.

Par rapport aux structures existantes ce type d'ouvrage a pour finalité l'interception de blocs d'énergie modérée dans des zones à forte contrainte d'emprise au sol.

L'étude menée est conduite suivant une approche multi-échelles : du matériau constitutif à l'ouvrage (Bertrand. 2006). Elle s'appuie sur des modélisations expérimentale et numérique en forte interaction, Les précédents travaux expérimentaux ont principalement concerné les matériaux constitutifs des cellules ainsi que le comportement sous chargement pseudo-statique de cellules seules (Fig. 2) (Lambert et al., 2004). Ces expérimentations ont notamment permis de modéliser par la méthode aux éléments distincts le comportement des cellules remplies de granulats sous sollicitations quasi-statiques (Gotteland et al., 2005a) (Bertrand et al., 2005).

Dans ce même contexte plusieurs auteurs se sont récemment intéressés au comportement sous impact de géomatériaux avec des expériences sur sol en place (Pichler et al., 2005), sur matériaux amortissants en couche (Montani Stoffel, 1998) ou sur des dalles en béton (Delhomme, 2005). D'autres se sont par ailleurs intéressés à l'effet du confinement par une enveloppe sur un géomatériau, principalement en abordant le comportement d'objets cylindriques soumis à chargement pseudo-statique parallèlement ou perpendiculairement à leur axe (Bathurst et Karpurapu, 1993); (lizuka et al., 2004).

L'article s'intéresse au comportement d'une géocellule de forme parallélépipède rectangle remplie de matériaux granulaires et soumise à un impact. En par- 
(a)

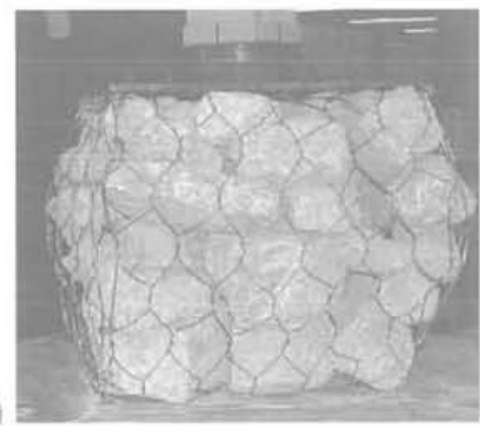

(b)
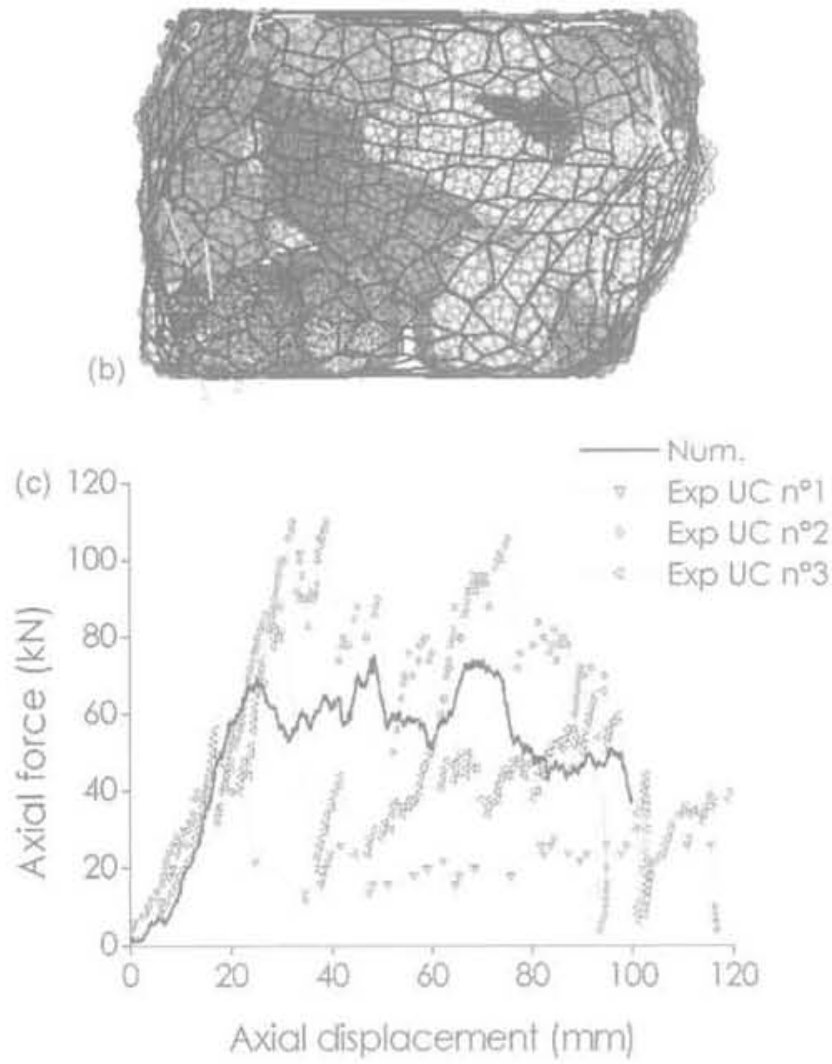

Fig. 2. Compression pseudo-statique d'une cellule non confinée (NC) : cellule expérimentale (a) et numérique (b), résultats expérimentaux et résultat numérique (c) (Gotteland et al., 2005a).

Quasi-static compression tests on unconfinec cell (NC): experiment (a) and modelling (b) experimental and numerical results (c) (Gotteland et al., 2005a).

ticulier, on évalue : l'influence du matériau de remplissage, de l'énergie cinétique de l'impactant et des conditions aux limites de la cellule sur sa réponse à l'împact. La réponse est évaluée en termes de résistance opposée à la pénétration de l'impactant et en termes de force transmise par la cellule. L'objectif est d'identifier les conditions pour une dissipation d'énergie maximale et une réduction des efforts transmis au support par la cellule. Les expérimentations réalisées sont présentées, les résultats discutés, puis comparés avec les simulations numériques.

\section{2}

\section{Matériel et méthodes}

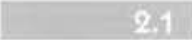

\section{Matériel}

Les géocellules étudiées sont constituées de cages de grillage métallique remplies de matériaux granulaires et usuellement nommées gabions. Pour des raisons de symétrie par rapport à l'axe de chargement, les cages utilisées sont cubiques et sont montées sans les tirants qui, habituellement, relient toute face exposée du gabion à son opposée et limitent les déformations latérales. Les cages utilisées sont des cubes de 500 et 1000 mm d'arête fabriquées à partir de grillage double torsion, c'est-àdire à maille hexagonale, dont la résistance à la rupture en traction dans le sens des torsades est de $51 \mathrm{kN} / \mathrm{m}$.

Les matériaux de remplissage sont soit des matériaux grossiers soit des matériaux fins. Les matériaux grossiers sont issus du concassage en carrière de calcaire urgonien de $58000 \mathrm{MPa}$ de module. Les granulats de remplissage des cellules de 500 et $1000 \mathrm{~mm}$ d'arète ont une granulométrie de 60-180 mm et 60$250 \mathrm{~mm}$, respectivement, en accord avec la norme francaise relative aux ouvrages en gabions (Afnor, 2004). Les matériaux fins sont le sable d'Hostun RF et un mélange de ce mème sable avec 30 \% en masse de déchiquetures de pneus (Fig. 3). Ce matériau compo-
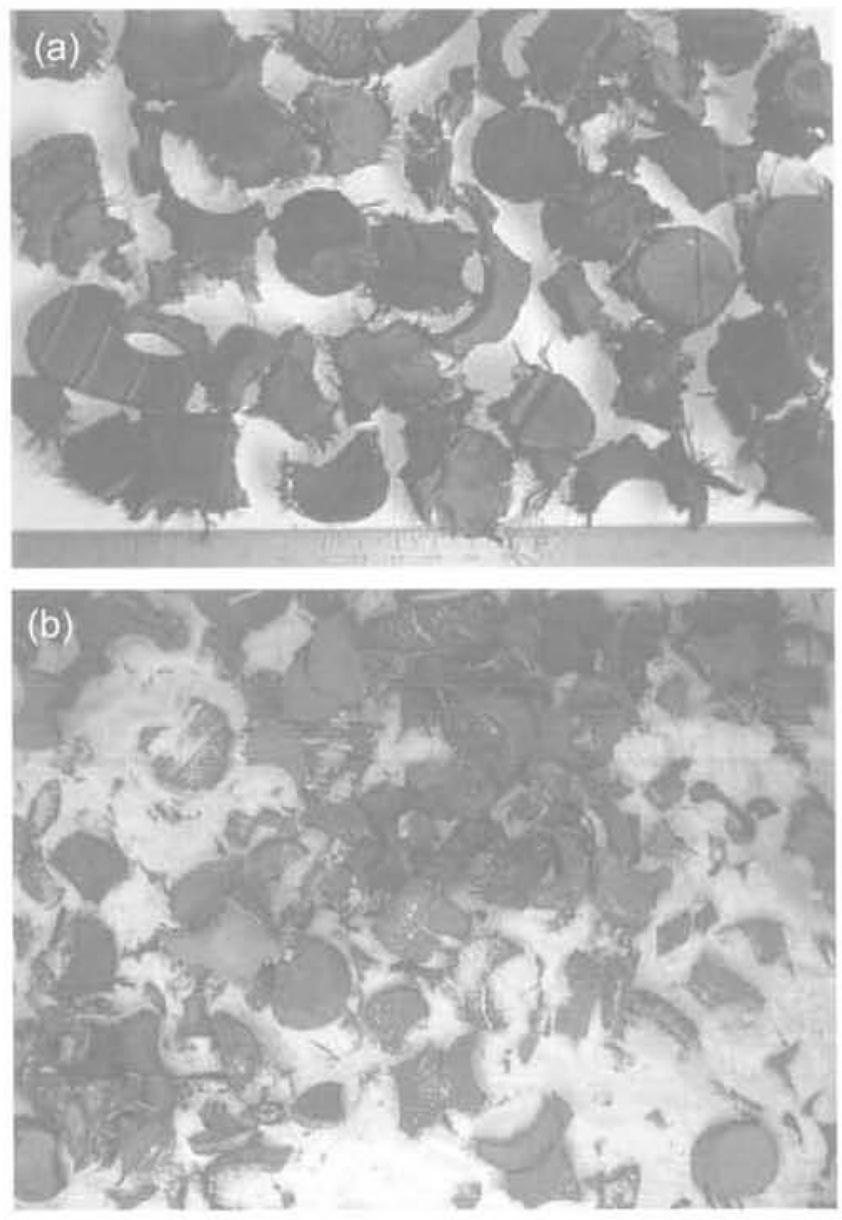

FIG 3 Pneus déchiquetés (a) et mélange (M) pneu-sable à $30 \%$ en masse de pneus (b). Shredded tyres (a) and tyre-sand mix (M) containing $30 \%$ by mass of tyres (b). 
site a été retenu pour ses caractéristiques mécaniques mais également à des fins de valorisation. La déchiqueture de pneus étudiée résulte du poinçonnement de pneus usagés non réutilisables et consiste en un mélange de copeaux dont $30 \%$ en masse sont des pastilles de forme circulaire de $25 \mathrm{~mm}$ de diamètre et le reste est de forme indéfinissable résultant de la méthode de poinçonnement. Un tel mélange de copeaux de pneu et de sable constitue un matériau composite renforcé et allégé (Gotteland et al., 2005b).

Les matériaux fins sont contenus dans la cage grillagée par l'intermédiaire d'une chaussette en géotextile non-tissé aiguilleté de polyester de $20 \mathrm{kN} / \mathrm{m}$ de résistance à la traction. Sur les faces latérales, cette enveloppe de contention est constituée d'une bande de géotextile se recouvrant sur la longueur d'une face. Ce chevauchement permet d'assurer la contention du matériau quelle que soit la déformation de la cellule.

Un coffre de confinement est utilisé lors du remplissage pour limiter la déformation des cages avant essai. Les granulats sont disposés manuellement « à plat ), de telle sorte que leur plus petite dimension soit suivant la verticale. Le sable est moyennement compacté. Les géocellules sont confectionnées sur le site expérimental afin d'éviter les phénomènes de compactage et de réarrangement liés aux vibrations durant le transport.

\section{Méthode}

Les sollicitations dynamiques sont appliquées sur les cellules par chute libre d'un impactant depuis des hauteurs de 4 à $7 \mathrm{~m}$ (Fig. 4). L'impactant est une coque sphérique en acier de $12 \mathrm{~mm}$ d'épaisseur remplie de béton fibré, de poids volumique proche de $30 \mathrm{kN} / \mathrm{m}^{3}$. Un fourreau permet de placer un accéléromètre triaxial en son centre de gravité.

Les cellules sont placées sur un socle en béton armé considéré indéformable reposant sur trois capteurs de force disposés en trépied sur une dalle en béton armée coulée sur le sol du site.

Compte tenu des objectifs de l'étude, l'instrumentation mise en cuvre vise à analyser la force opposée par la cellule à la pénétration par l'impactant et la force transmise par la cellule, sur toute la durée de l'impact. La première donnée est déduite de la mesure de l'accélération de l'impactant. La seconde est obtenue par mesure de l'effort transmis par le socle à la dalle, faute de pouvoir mesurer directement la force transmise par la cellule au socle, notamment dans le cas de cellules remplies d'éléments grossiers. Le traitement de cette donnée se fait par comparaison de réponse dans les différentes configurations d'essai.

Après essai, on mesure le périmètre de la cellule à mi-hauteur dont on déduit la variation relative par rapport au périmètre initial, $\Delta \mathrm{P}(\%)$. On mesure également l'enfoncement de l'impactant dans la cellule ; c'est la distance parcourue par l'impactant entre le point de contact avec la cellule et le point d'immobilisation.

Lors de l'impact, les faces latérales des cellules sont soit laissées libres de se déformer (essais non confinés $\mathrm{NC}$ ) (Fig. 5a) soit maintenues fixes par une structure de confinement rigide (essais confinés - C) (Fig. 5b). Ces deux situations constituent les cas extrêmes des conditions aux limites attendues au sein de l'ouvrage : la réponse des cellules sera modifiée par l'interaction avec les cellules contiguës, qu'elles soient espacées ou non. Les conditions expérimentales sont donc simplifiées pour faciliter la compréhension phénoménologique du comportement des géocellules composites impactées. Cette simplicité limite la possibilité d'extrapolation directe au comportement des cellules dans l'ouvrage mais est également nécessaire à l'accompagnement de la démarche de modélisation numérique menée en parallèle.

\section{3}

\section{Résultats expérimentaux}

Des cellules de taille et de matériaux de remplissage différents ont été soumises à impacts d'énergie variable, avec ou sans confinement latéral (Tableaú 1). L'identifiant de chaque essai mentionné en première colonne du tableau fait pleinement référence aux conditions d'essai. Les colonnes de droite du tableau présentent les principaux résultats ; ils seront abordés au paragraphe suivant.
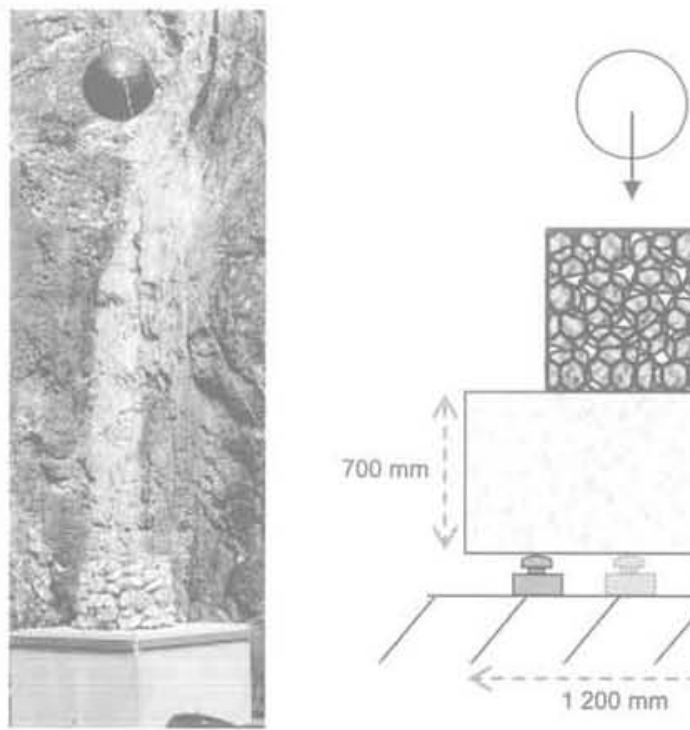

Impactant $(250 \mathrm{~kg}, \varphi=540 \mathrm{~mm})$ avec accélèromètre $(+/-500 \mathrm{~g})$

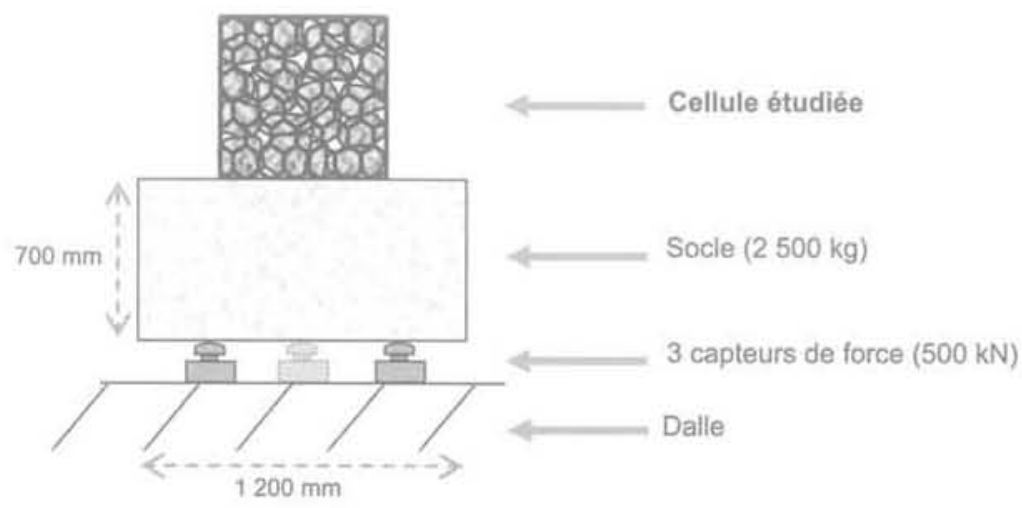



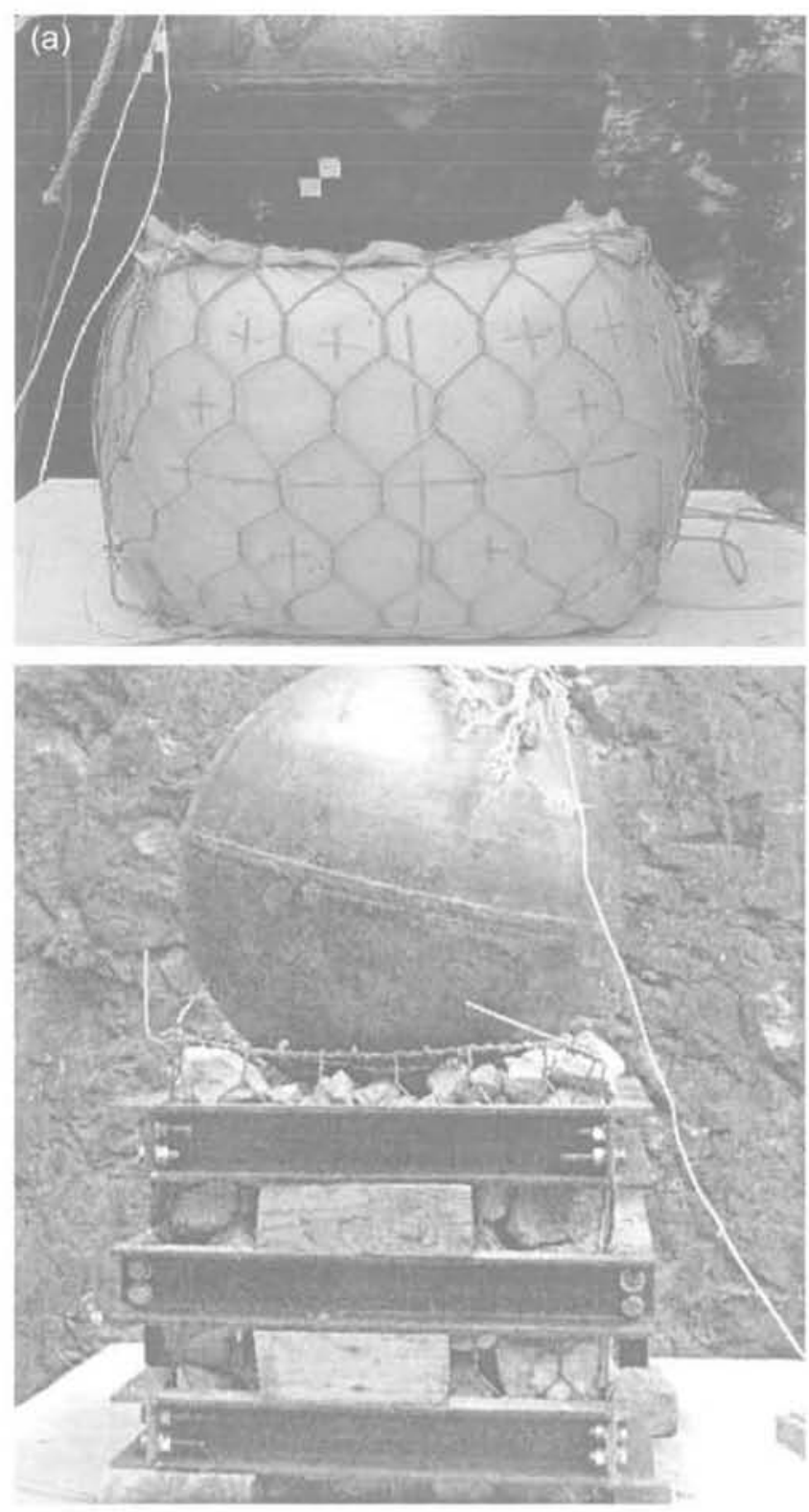

FG. 5 Cellule de $500 \mathrm{~mm}$ remplie de sable non confinée (a) et cellule de $500 \mathrm{~mm}$ remplie de granulats confinée (b), après impact.

$500 \mathrm{~mm}$ in height unconfined sand cell (a) and $500 \mathrm{~mm}$ in height gravel contined cell (b) after impact.

Lors de la chute, puis de l'impact, la rotation de l'impactant est faible. Le point d'impact est bien centré sur la cellule. Lors de l'impact, la cellule subit un affaissement conjointement à un gonflement latéral, sauf dans le cas de cellules confinées (Fig. 6). Sur les cellules de $500 \mathrm{~mm}$ remplies de granulats, l'impact provoque de nombreuses fracturations des granulats et conduit à l'ouverture et l'éjection d'agrafes de liaison des panneaux de grillage pour les énergies d'impact élevées. Sur les cellules de $500 \mathrm{~mm}$ remplies de matériaux fins, on observe un glissement de l'ordre de $100 \mathrm{~mm}$ sur la zone de recouvrement du géotextile de contention latérale, ainsi qu'un allongement également de l'ordre de $100 \mathrm{~mm}$ suivant le périmètre de la cellule, traduisant une sollicitation mécanique du géotextile. En fin d'impact sur les cellules confinées et sur les cellules de $500 \mathrm{~mm}$ remplies de sable (S) et de mélange pneu-sable (M) non confinées l'impactant remonte, avec rebond important dans le premier cas. Ce n'est pas le cas pour
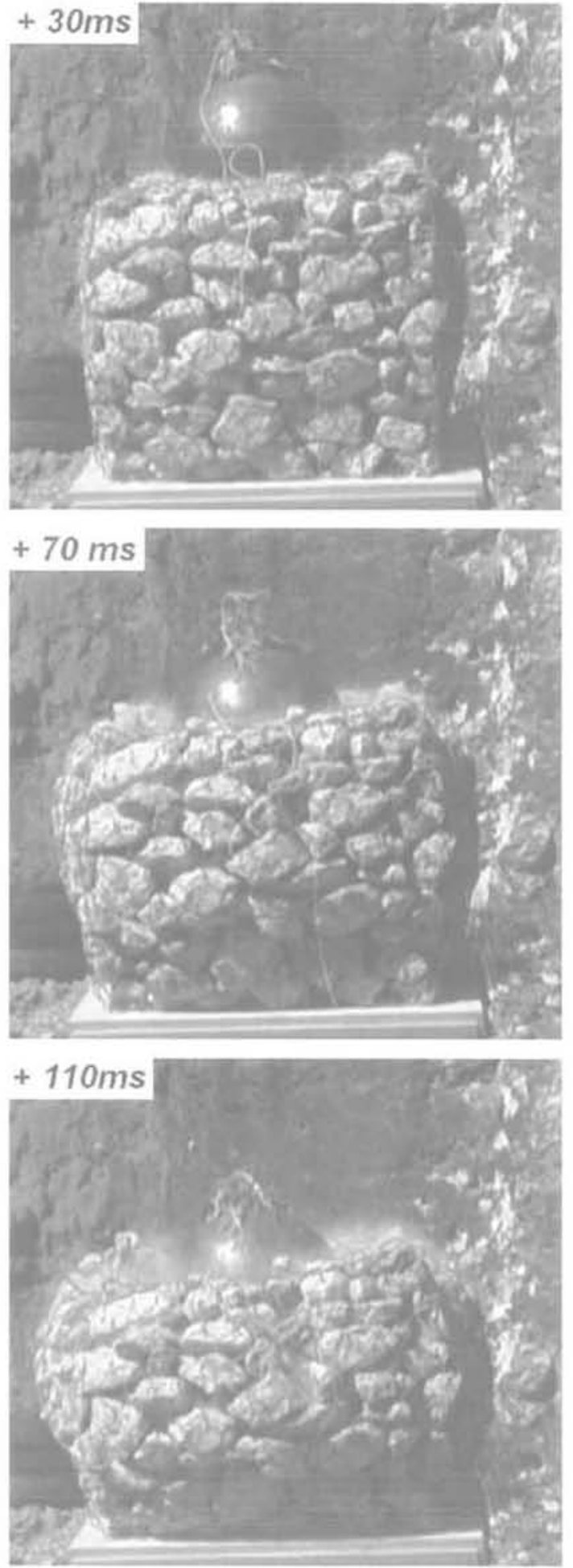

FIG.6 Impact sur une cellule de $1000 \mathrm{~mm}$ remplie de granulats, non confinée, à 3 instants après contact (essai 1000 G NC 7,4). Impact on a $1,000 \mathrm{~mm}$ in height cell filled with gravel at 3 times after contact (test 1000_G_NC_7,4). 
TABLEAU1 Caractéristiques des essais et principaux résultats. Tests conditions and main results.

\begin{tabular}{|c|c|c|c|c|c|c|c|c|}
\hline Identifiant de l'essaj" & $\begin{array}{l}\text { Masse de la cellule } \\
\qquad(\mathrm{kg})\end{array}$ & $\begin{array}{l}\text { Bnergie cinétique } \\
\text { du bloc avant impact } \\
\text { (k.J) }\end{array}$ & $\begin{array}{l}F_{\text {imp }} \\
(\mathrm{kN})\end{array}$ & $\begin{array}{l}\mathrm{F}_{\text {AnE }} \\
(\mathrm{kN})\end{array}$ & $\begin{array}{l}\text { Att: } \\
(-)\end{array}$ & $\begin{array}{l}\text { Enf: } \\
\text { (mm) }\end{array}$ & (ms) & $\begin{array}{l}\Delta \mathrm{P} \\
(\%)\end{array}$ \\
\hline 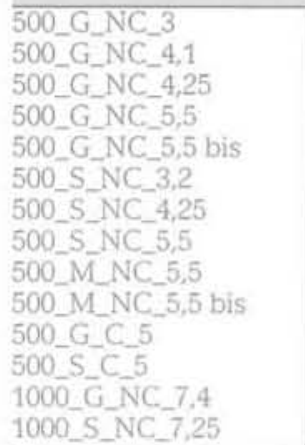 & $\begin{array}{l}208 \\
205 \\
215 \\
215 \\
215 \\
200 \\
205 \\
202 \\
190 \\
190 \\
204 \\
206 \\
1538 \\
1441\end{array}$ & $\begin{array}{l}7,4 \\
10,1 \\
10,4 \\
13,5 \\
13,5 \\
7,8 \\
10,4 \\
13,5 \\
13,5 \\
13,5 \\
12,3 \\
12,3 \\
18,1 \\
17,9\end{array}$ & \begin{tabular}{|c|}
68 \\
76 \\
112 \\
127 \\
144 \\
43 \\
60 \\
81 \\
48 \\
74 \\
242 \\
346 \\
128 \\
86 \\
\end{tabular} & $\begin{array}{c}47 \\
53 \\
52 \\
77 \\
43 \\
27 \\
37 \\
46 \\
39 \\
40 \\
377 \\
601 \\
97 \\
75 \\
\end{array}$ & $\begin{array}{l}0,7 \\
0,7 \\
0,5 \\
0,6 \\
0,6 \\
0,6 \\
0,6 \\
0,6 \\
0,8 \\
0,5 \\
1,6 \\
1,7 \\
0,8 \\
0,9\end{array}$ & $\begin{array}{l}300 \\
330 \\
390 \\
330 \\
400 \\
210 \\
220 \\
210 \\
230 \\
260 \\
80 \\
60 \\
630 \\
510\end{array}$ & $\begin{array}{l}\cong 110 \\
\cong 105 \\
\cong 90 \\
\cong 100 \\
\cong 100 \\
\cong 90 \\
\cong 90 \\
\cong 80 \\
\cong 65 \\
\cong 65 \\
\cong 26 \\
\cong 22 \\
\geq 150 \\
\geqq 120\end{array}$ & $\begin{array}{c}42 \\
35 \\
39 \\
- \\
46 \\
13 \\
13 \\
16 \\
12 \\
19 \\
- \\
- \\
25 \\
9\end{array}$ \\
\hline
\end{tabular}

* L'identifiant fait successivement référence à la taille de la cellule ( 500 ou $1000 \mathrm{~mm}$ ), au matériau de remplissacie (G pour granulats, S pour sable et $\mathrm{M}$ pour mélange de sable avec $30 \%$ en masse de pneus), aux conditions aux limites (C pour confiné et NC pour non confiné) et à la hauteur de chute, en mètres.
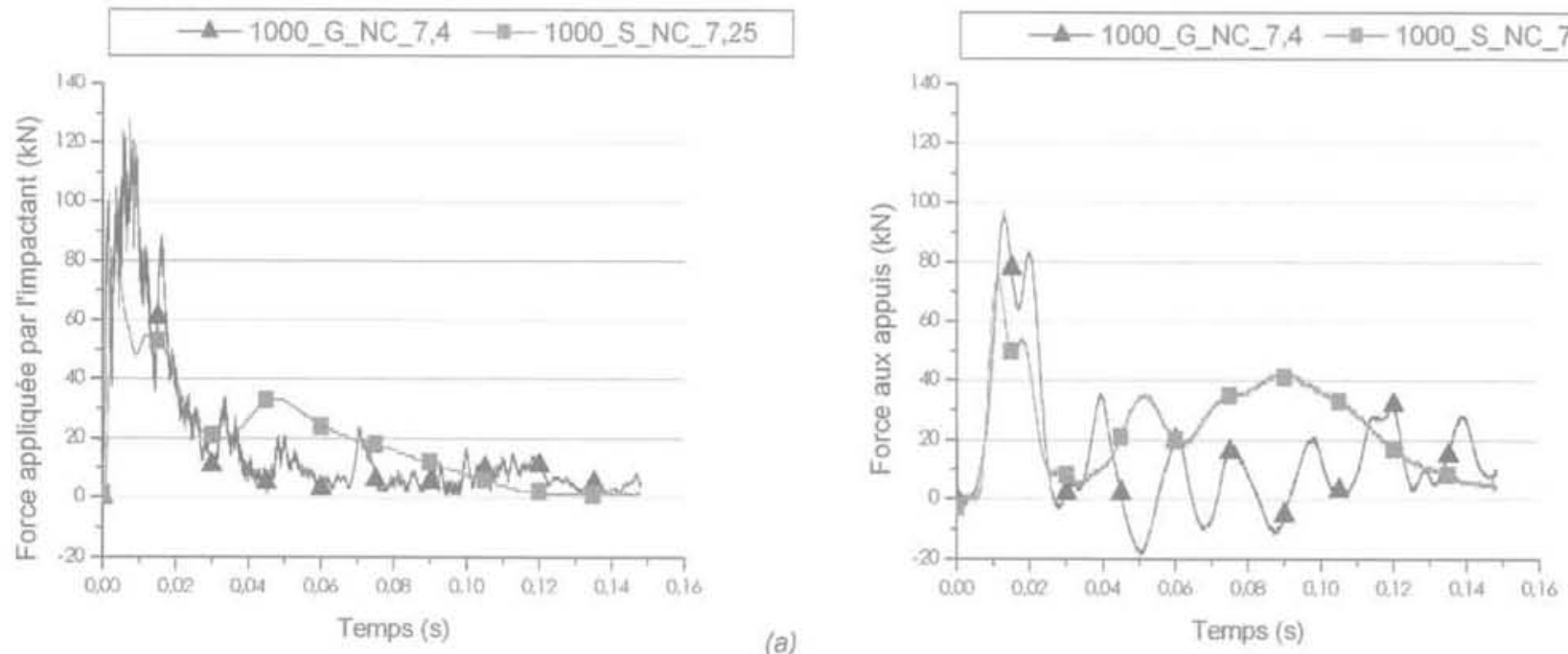

(a)

Temps (s)

FG. 7 Force appliquée par l'impactant sur la cellule (a) et force transmise aux appuis (b) pour les cellules de $1000 \mathrm{~mm}$ non confinées remplies de granulats et de sable.

Force applied by the boulder on the cell (a) and force on the load transducers (b) for $1,000 \mathrm{~mm}$ in height unconfined cells filled with gravel and sand.
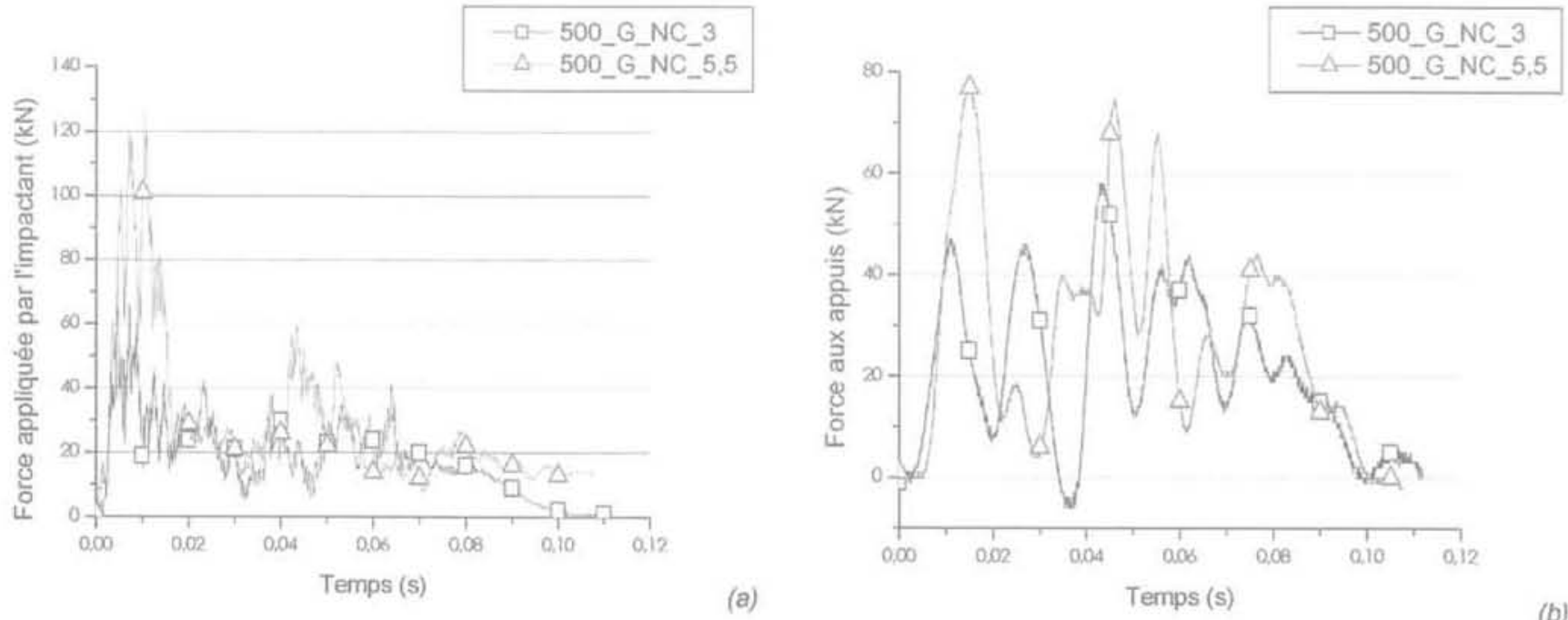

(b)

FG. 8 Force appliquée par l'impactant sur la cellule (a) et force aux appuis (b) lors de l'impact de cellules de $500 \mathrm{~mm}$ remplies de granulats, non confinées, pour 2 hauteurs de chute.

Force applied by the boulder on the cell (a) and force on the load transducers (b) during impacts on $500 \mathrm{~mm}$ in height unconfined cells for 2 boulder falling heights. 

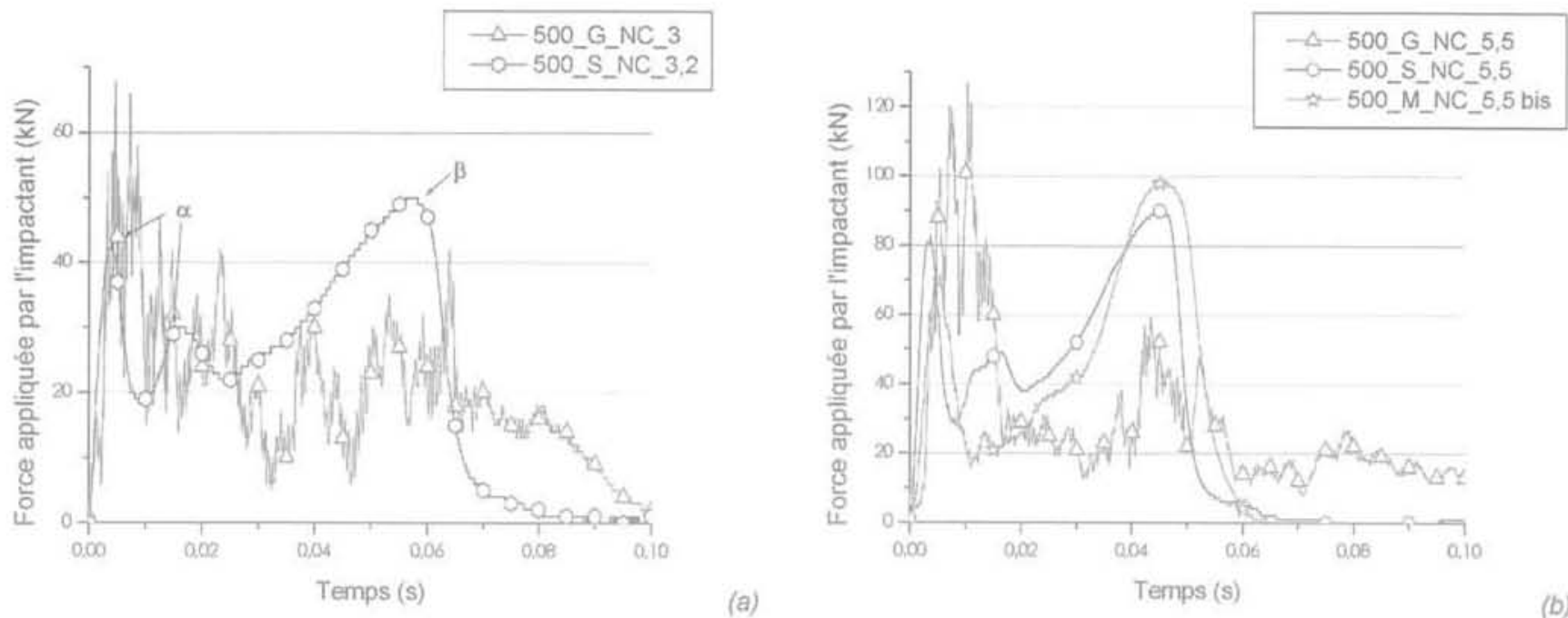

nG.9 Influence du type de matériau de remplissage (granulats/sable/mélange pneu-sables) sur la force appliquée par l'impactant pour deux hauteurs de chute : $3 \mathrm{~m}$ (a) et $5,5 \mathrm{~m}$ (b).

Influence of the type of filling material (gravel/sand/tyre-sand mixture) on the force applied by the boulder for two boulder falling height : $3 \mathrm{~m}(\mathrm{a})$ and $5.5 \mathrm{~m}(\mathrm{~b})$.

les cellules remplies de granulats et non confinées. Pour les cellules remplies de mélange pneu-sable (M), ce rebond n'est pas vertical, du fait de la difficulté d'assurer lors de la mise en cuvre une parfaite homogénéité de ce matériau composite. De fait, en fín d'impact, l'impactant est éjecté alors que dans les autres situations il s'immobilise sur la cellule.

A partir de l'accélération de l'impactant on obtient la force appliquée par l'impactant sur la cellule comme étant le produit de la masse de l'impactant par son accélération. Cette force est équivalente à la force opposée par la cellule à la pénétration par l'impactant (ex. Fig. 7a), La valeur de la force au premier pic est définie comme étant la force d'impact, $\mathrm{F}_{\text {imp. }}$. Ce pic est toujours observé dans les 30 millisecondes après contact.

La somme des trois mesures de force donne la force résultante transmise aux appuis. Ces mesures de force sont souvent perturbées par les oscillations du système mécanique (ex. Figs. $7 \mathrm{~b}$ et $8 \mathrm{~b}$ ). Néanmoins la valeur de cette force au premier pic, $F_{\text {Apir }}$, sera directement comparée à la force d'impact, $F_{\text {mp' }}$ elle aussi mesurée en début d'impact.

Dans certaines configurations $\mathrm{F}_{\text {Imp }}$ et $\mathrm{F}_{\text {Apic }}$ ne sont pas les valeurs maximales des forces sur la durée de l'impact. Ces maximums peuvent être atteints pour des temps après contact assez importants, donc pour de grandes déformations de la cellule (Figs. 8 b et 9). Le comportement des cellules très déformées n'étant pas représentatif de celui attendu au sein de l'ouvrage n'a pas été retenu. En effet, les conditions expérimentales en lâché vertical s'écartent des conditions réelles d'utilisation des cellules en interaction dans l'ouvrage. La cellule est posée sur un support fixe indéformable et les conditions aux limites latérales sont des conditions extrêmes par rapport à celles attendues au sein de l'ouvrage. D'autres essais de performance sont en cours et devraient permettre le lien avec le fonctionnement en situation réelle.

L'atténuation est définie comme le rapport des deux forces précédentes, Att. $=\mathrm{F}_{\text {Apic }} / \mathrm{F}_{\text {imp }}$.

La durée de l'impact, $t_{\text {imp }}$, est déduite des mesures d'accélération.
Ces résultats, ainsi que les valeurs d'enfoncement, Enf, et de variation relative de périmètre, $\Delta \mathrm{P}$, sont reportés sur les colonnes de droite du tableau $\mathrm{L}$.

Dans le cas des cellules de $500 \mathrm{~mm}$ non confinées remplies de sable (S) et de mélange pneu-sable (M), les valeurs d'enfoncement et de périmètre mesurées sont inférieures aux valeurs maximales atteintes au cours de l'impact, du fait du rebond de l'impactant, donc de la décompression de la cellule.

L'ímpossibilité de reproduire d'un essai à l'autre la mise en cuvre des granulats et des mélanges pneusable introduit une variabilité des valeurs de force obtenues pour les cellules remplies de ces matériaux.

Les mesures d'accélération peuvent être utilisées pour déterminer l'enfoncement de l'impactant en tout instant de l'impact, permettant notamment de présenter les courbes de force appliquée par l'impactant en fonction de son déplacement. Le calcul conduit cependant à des incertitudes non négligeables sur les valeurs d'enfoncement. A défaut, des valeurs indicatives d'enfoncement sont données pour certains instants particuliers.

\section{Discussion}

La géo-cellule est un système composite constitué d'une enveloppe et d'un matériau de remplissage granulaire. Le comportement observé dépend des caractéristiques de chacun des constituants et de leur interaction. La compréhension de ce comportement est abordée à travers une discussion sur l'influence de différents paramètres et en particulier : le matériau de remplissage, les conditions aux limites et la hauteur de chute (énergie d'impact).

\section{1}

\section{Influence du matériau de remplissage}

Les caractéristiques du matériau de remplissage ont une influence sur la forme des courbes et sur les valeurs particulières. 
Dans le cas des cellules remplies de granulats, les variations brutales de force sur l'impactant au cours de l'impact (Figs. 7a et 8a) s'expliquent par la nature grossière du matériau de remplissage. La transmission des efforts au sein d'une assemblée granulaire grossière se fait par le biais de chaines de force, supportées par des colonnes de granulats. Pour les contacts, on distingue une phase forte entre les granulats mobilisés dans les colonnes, d'une phase faible entre les colonnes et les autres granulats, contribuant à la stabilité des colonnes (Radjai et al., 1998). Lorsqu'une de ces chaines rompt, soit par déplacement d'un des granulats de la colonne (Oda et al., 1982) soit par rupture d'un de ces éléments (Tsoungui et al., 1999), la force reprise par l'assemblée décroît brusquement jusqu'à ce qu'une nouvelle colonne soit mobilisée. Les paramètres influant sur ces variations brutales sont notamment la taille moyenne des éléments, leur forme et leur arrangement initial. Comparativement, les courbes relatives aux cellules sable (S) et pneu-sable (M) sont plus lisses (Figs, 7a et 9).

L'utilisation de sable en lieu et place des granulats, pour les cellules de $500 \mathrm{~mm}$ comme pour les cellules de $1000 \mathrm{~mm}$, conduit à une réduction : des valeurs des forces d'impact et aux appuis, $\bar{F}_{\text {imi }}$ et $F_{\text {Anic }}$ des valeurs d'enfoncement de l'impactant et de la durée d'impact (Tableau 1). En début d'impact, c'est-à-dire sur les 20 premières millisecondes, la force opposée à la pénétration de l'impactant est sensiblement plus faible pour une cellule remplie de sable comparativernent à une cellule remplie de granulats ; en particulier, le premier pic est plus étroit et est suivi d'un deuxième pic moins prononcé (point $\alpha$, Fig. 9a). Au-delà des effets dynamiques, ceci s'explique par la plus faible « rigidité apparente » du volume de matériau de remplissage effectivement sollicité pendant cette première phase de l'impact. Vers la fin de l'impact, la force opposée à la pénétration dé l'impactant par une cellule remplie de sable augmente de manière marquée (point $\beta$, Fig, 9a). Ce pic se manifeste à l'enfoncement maximal lors de l'impact et marque le début de remontée de l'impactant. Cette forme de courbe se distingue nettement des courbes classiquement obtenues lors d'impacts sur couches de matériaux granulaires fins (p. ex. Montani Stoffel, 1998). Cette augmentation est due à la mobilisation effective de l'enveloppe constituée du grillage et du géotextile, appliquant sur le matériau de remplissage une contrainte de confinement croissante. Cette mobilisation n'est effective que lorsque la cellule est très déformée et que sa forme, initialement cubique, a changé. En présence de tirants l'enveloppe serait mobilisée plus tôt, puisque ceux-ci ont pour effet de limiter la déformation latérale des cellules. Il en serait de méme si la cellule était initialement cylindrique.

Pour les cellules remplies de granulats, de $500 \mathrm{~mm}$ et de $1000 \mathrm{~mm}$, cet accroissement de force en fin d'impact n'apparait pas, bien que la déformation après impact soit plus importante. La déformation peut être évaluée à partir des enfoncement et variation de longueur du périmètre mesurés (Tableau 1). Notons que cette variation traduit le niveau de sollicitation de l'enveloppe. Cette différence de comportement en fin d'impact peut être expliquée par les différences de configurations entre les deux types de cellules granulats et sable. Ces différences tiennent à la fois à l'enveloppe et au matériau de remplissage.

L'enveloppe des cellules remplies de granulats est uniquement constituée du grillage alors que celle des cellules sable inclue également le géotextile. Or, il est avéré que le géotextile est effectivement sollicité en traction. Il contribue donc à l'effet de confinement par l'enveloppe. Cependant, compte tenu du rapport de 10 des modules d'élongation du géotextile et du grillage en faveur du grillage, le surcroit de confinement lié à la présence du géotextile est faible. L'absence de géotextile sur les cellules granulats ne peut expliquer seule la différence de comportement observée en fin d'impact entre les deux types de cellules.

La différence de matériau de remplissage peut être à l'origine d'une modification de l'influence de l'enveloppe sur la réponse de la cellule. Il semble que l'existence de chaînes de force au sein de l'assemblée granulaire réduise l'influence de l'effet de confinement latéral. Les chaines sont particulièrement stables du fait de la forme des granulats : l'enveloppe a peu d'effet sur leur stabilité. Pour les cellules de $500 \mathrm{~mm}$, la distance entre impactant et socle est inférieure à $300 \mathrm{~mm}$ au-delà de $40 \mathrm{~ms}$ après le contact. représentant environ trois granulats. Les colonnes constituées d'un si faible nombre de granulats sont moins sensibles à toute action latérale, tel que l'effet de confinement par l'enveloppe. Pour les cellules de $1000 \mathrm{~mm}$, pour lesquelles le rapport de taille initial entre cranulats et cellule est de 8, l'enfoncement est moindre et le nombre minimum de granulats mobilisés dans les colonnes est plus élevé. Pour ces cellules, on peut supposer que l'effet de confinement ne peut se propager au sein de l'assemblée granulaire du fait de la réduction du nombre de contacts de la phase faible, phénomène résultant des fortes déformations subies par la cellule (Radjai et al., 1999), Il apparaît donc que c'est la nature granulaire grossière du matériau de remplissage qui explique cette différence de comportement entre cellule granulats et cellule sable.

Par ailleurs, au-delà du temps de $20 \mathrm{~ms}$ après contact, la force que peut reprendre une cellule remplie de granulats est limitée par la résistance en compression des granulats mobilisés dans la phase forte. En effet, la force transitant dans une colonne de granulats ne peut dépasser la résistance maximale du plus faible de ses éléments. Chaque colonne se comporte ainsi en fusible et la force opposée au déplacement de l'impact est limitée par le nombre de colonnes mobilisées et les caractéristiques des granulats constitutifs.

Ainsi, ce sont les caractéristiques du matériau de remplissage qui gouvernent le comportement de la cellule en petites déformations, l'enveloppe n'étant pas ou peu sollicitée. Par contre, en grandes déformations, la force résistante à l'impact opposée par une cellule non confinée dépend à la fois des caractérístiques de l'enveloppe et des caractéristiques du matériau de remplissage.

L'incorporation de déchiquetures de pneus dans le sable se traduit principalement par une réduction de la durée de l'impact et une augmentation de l'enfoncement. Les valeurs des forces d'impact et aux appuis, $\mathrm{F}$ et $\mathrm{F}_{\text {an }}$, sont sensiblement réduites. En fait, la plus faible résistance opposée par la cellule remplie de mélange pneu-sable en début d'impact conduit à un plus fort enfoncement en grandes déformations. Ce plus fort enfoncement engendre une plus forte sollicitation de l'enveloppe et donc une augmentation de la valeur de force au pic $\beta$ (Fig. 9 b). Au final, le temps d'impact est sensiblement réduit.

Globalement, l'accélération subie par l'impactant au cours de l'impact avec une cellule sable ou une cellule pneu-sable est plus uniforme et en movenne plus élevée qu'avec une cellule granulats, à énergie d'impact identique. La force transmise aux appuis en début 
d'impact est plus faible. Dans ces conditions d'essai une cellule remplie de sable (S) ou de mélange pneusable $(\mathrm{M})$ répond mieux aux attentes d'atténuation des efforts transmis à la partie à l'aval.

\section{2}

\section{Influence des conditions aux limites}

Le confinement (C) affecte le comportement de la cellule par une augmentation de la valeur de force d'impact, $F_{\text {imp }}$ et par une réduction de la durée de l'impact d'un rapport 4 par rapport à un essai non confiné (NC) (Fig. 10). Il conduit à une augmentation de la valeur de force transmise aux appuis par rapport à la valeur de force appliquée. Il conduit, par ailleurs, à un plus grand nombre de fracturations des granulats.

Le fait que l'atténuation soit supérieure à 1 (Tableau I) apparaît étonnant mais peut s'expliquer par une amplification des efforts induits par les ondes de choc produits par les frontières latérales rigides.

Contrairement à la situation sans confinement, on constate que la force d'impact et la force transmise par la cellule remplie de sable sont supérieures à celles de la cellule remplie de granulats. Cette différence de comportement s'interprète dans la mesure où une cellule remplie de sable confinée est moins compressible qu'une cellule remplie de granulats confinée, du fait de la possibilité de fracturation des granulats. Dans une cellule confinée, le réarrangement du matériau de remplissage est restreint, voire impossible, et la seule déformation possible est celle liée à la compression du matériau de remplissage. En fait, la restriction de mobilité du matériau de remplissage conduit à l'augmentation de la force opposée à la pénétration de l'impactant ayant une vitesse donnée. Dans le cas des granulats, cette augmentation de force conduit à leur dégradation par fracturation à partir d'une certaine valeur de force. Pour ces cellules, on atteint donc un seuil de résistance contrairement aux cellules remplies de sable et le temps nécessaire pour arrêter l'impactant en est augmenté. En condition confinée (C), une cellule remplie de granulats atténue ainsi plus les forces qu'une cellule remplie de sable.

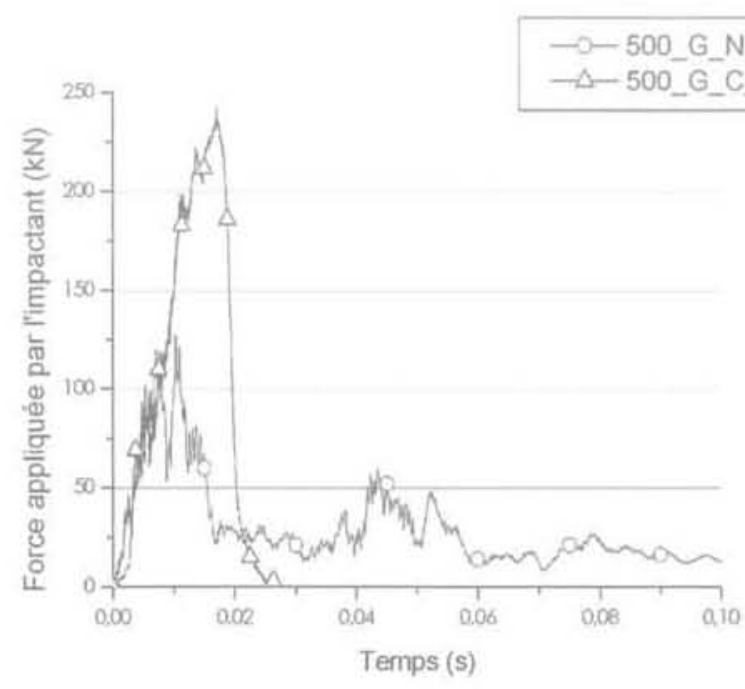

(a)
L'extrapolation de ces résultats au comportement des cellules dans l'ouvrage reste confrontée au problème des conditions aux limites. Pour le déplacement des faces latérales des cellules, il sera limité dans l'ouvrage par la présence des autres cellules, qu'elles soient en contact direct ou légèrement espacées. Ainsi, les conditions aux limites des cellules dans l'ourvrage seront intermédiaires entre les deux types de conditions aux limites testées (C ou NC). En particulier, à fortes déformations il y aura effectivement interaction entre la cellule impactée et les cellules contiguës. Dans les premiers instants, la cellule se déformant très peu latéralement les conditions aux limites latérales influent peu sur le comportement de la cellule. Pour la condition de support, lors des expérimentations réalisées les cellules reposent sur un support rigide alors qu'au sein de l'ouvrage la face opposée à la face impactée sera au contact d'une autre cellule, déformable. De telles configurations d'essais sont planifiées.

\section{4,3}

\section{Influence de la hauteur de chute, $\mathrm{Hc}$}

Pour les cellules de $500 \mathrm{~mm}$ non confinées remplies de sable ou de granulats, les forces d'impact et aux appuis. $F_{\text {imp }}$ et $F_{\text {Apic }}$ montrent une dépendance à la hauteur de chute, donc à l'énergie cinétique de l'impactant avant contact avec la cellule (Fig. 11). Il en est de même pour I'enfoncement sur cellules de $500 \mathrm{~mm}$ remplies de granulats, ce qui est cohérent avec le plus grand nombre de fracturations observées. Par contre, l'énergie d'impact semble avoir peu d'influence sur la réponse de ces cellules aux grandes déformations en termes de force opposée à la pénétration de l'impactant : les courbes relatives aux 4 impacts sur cellules de $500 \mathrm{~mm}$ remplies de granulats non confinées sont très similaires au-delà de $50 \mathrm{~ms}$, soit pour des enfoncements supérieurs à $250 \mathrm{~mm}$, marquant un plateau à environ $20-30 \mathrm{kN}$ avant de diminuer (Fig. 8), Il semble que pendant les premiers instants l'énergie soit transmise à la cellule par des phénomènes collisionnels, donc fortement dépendant de l'énergie cinétique de l'impactant. Puis, l'énergie est principalement dissipée par fracturation des granulats constitutifs des chaines de force, jusqu'à immobilisation de l'impactant.

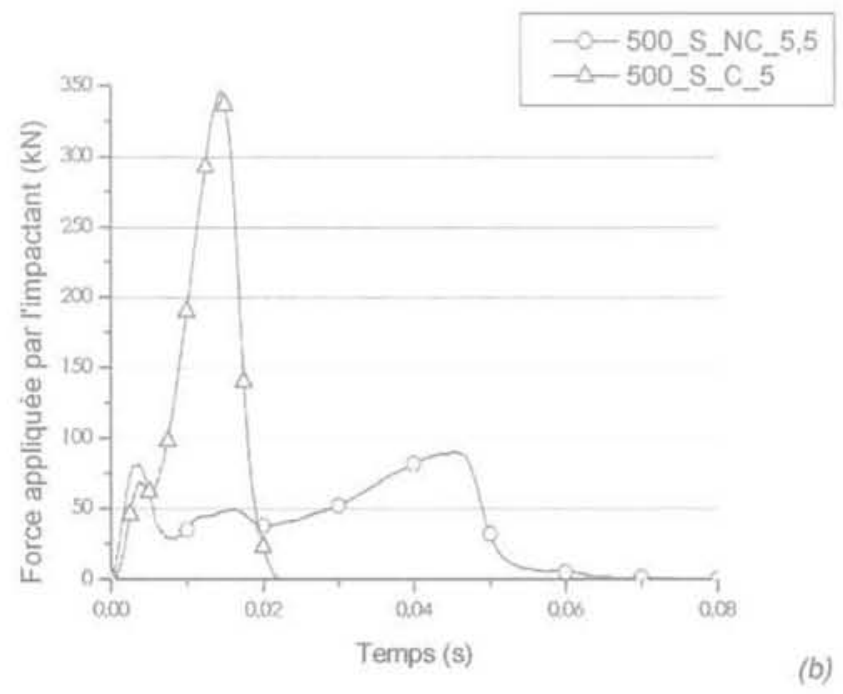

FG. 10 Influence des conditions aux limites (confiné/non confiné) sur la réponse des cellules remplies de granulats (a) et de sable (b) en termes de force appliquée par l'impactant.

influence of cell boundary conditions (confined C/unconfined NC) on the response of cells filled with gravels (a) and sand (b) in terms of force applied by the boulder. 
Dans le cas des cellules remplies de sable, la hauteur de chute modifie la réponse de la cellule en grandes déformations par une forte augmentation du pic $\beta$ (Fig. 9), traduisant une plus forte mobilisation de l'enveloppe.

\section{5}

\section{Modélisation numérique}

La modélisation numérique dans le domaine du génie civil fait largement appel à la méthode aux èléments finis. Cette méthode est en effet bien adaptée lorsque les matériaux sont assimilables à des matériaux continus. Cependant, elle se révèle moins performante lorsqu'il s'agit de modéliser une sollicitation de type impact localisé sur des matériaux grossiers, ou sur un ouvrage constitué de géocellules composites. L'approche discrète apparait mieux adaptée.

Les géocellules remplies de granulats ont ainsi été modélisées en trois dimensions par la méthode aux éléments distincts (DEM : discrete element method). Cette méthode permet de traiter le milieu comme un assemblage de corps distincts, prenant ici une forme sphérique (Bertrand, 2006). Les interactions entre ces sphères sont définies par des lois de contact locales, dont dépend le comportement macroscopique du milieu modélisé. Cette méthode permet également de gérer dans un même environnement de calcul les deux types de matériaux constitutifs des cellules l'enveloppe grillagée et le matériau de remplissage granulaire grossier.
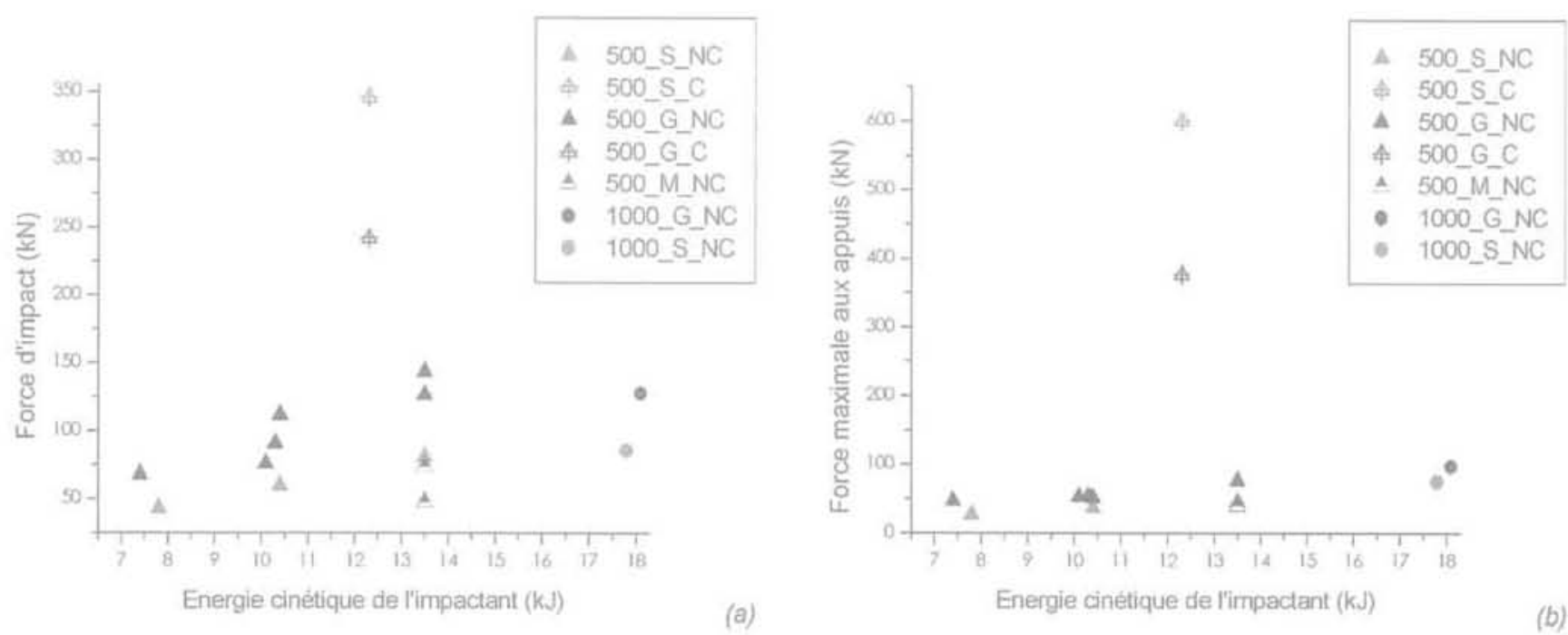

HG 11 Force d'impact, $F_{\text {imp }}$, (a) et force aux appuis au $1^{\text {er }}$ pic, $F_{\text {thic }}$ (b) pour tous les essais, en fonction de l'énergie cinétique de l'impactant.

Impact force, $F_{\text {my }}$ (a) and force on the load transducers, $F_{\text {spe }}$ (b) for all the tests, according to the kinetic energy of the boulder.
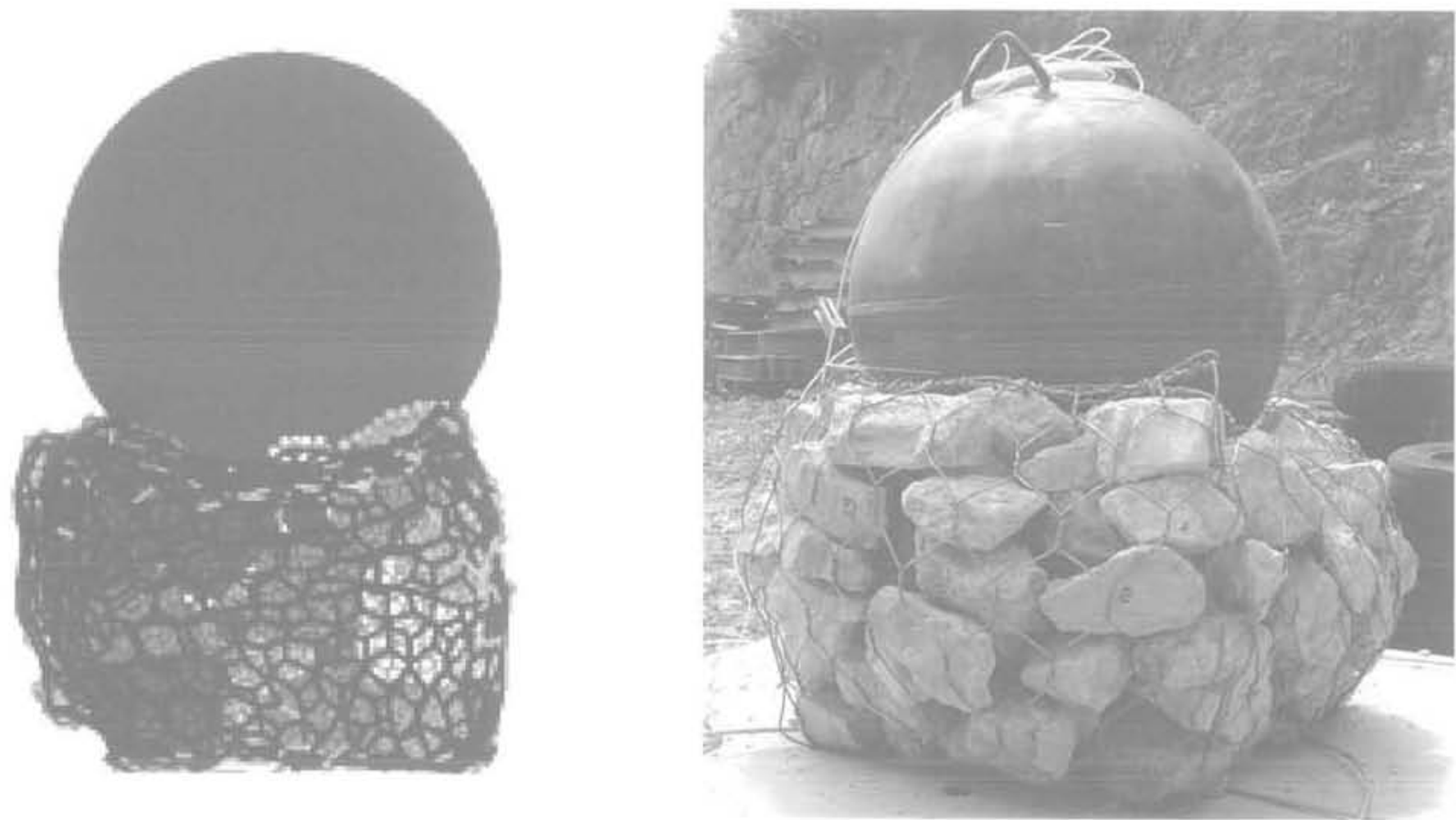

FIG.12 Cellule de $500 \mathrm{~mm}$ remplies de granulats après impact : numérique (a) et expérimental (b). $500 \mathrm{~mm}$ in height gravel cell after impact: simulation (a) and experimental (b). 

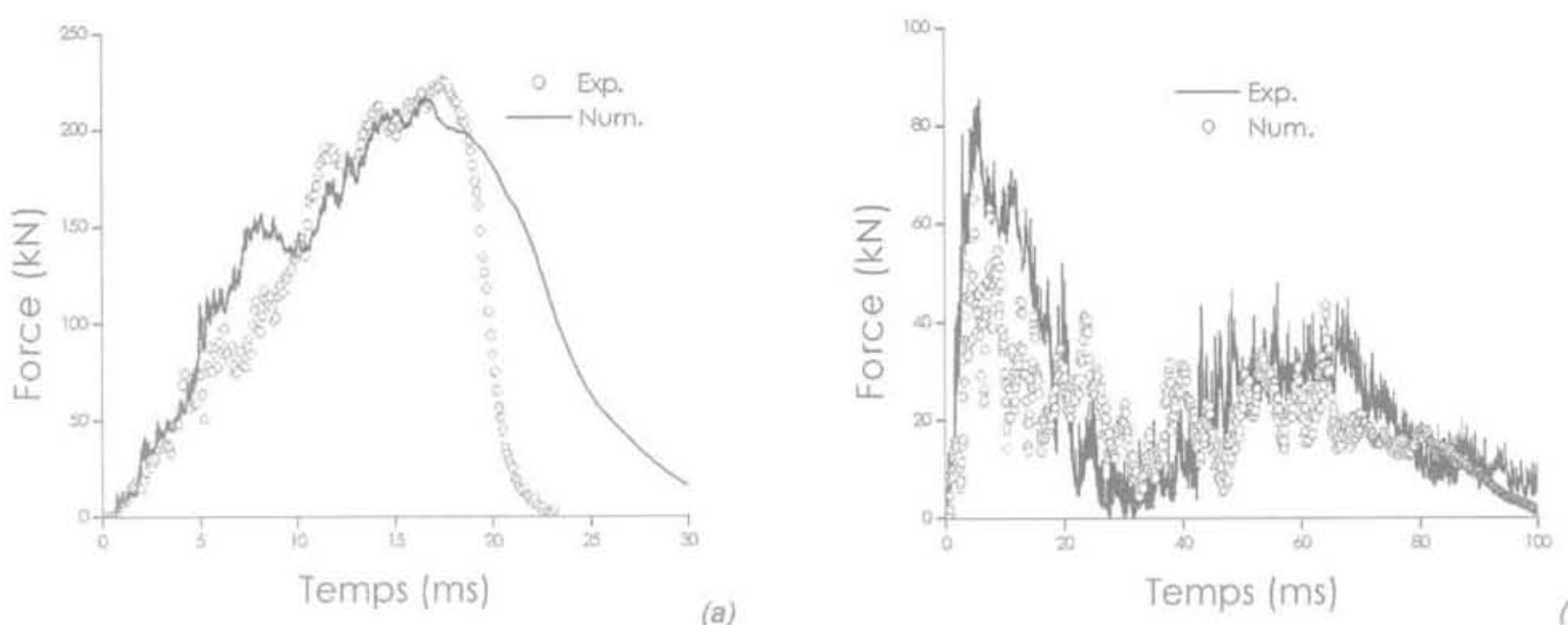

FIG. 13 Comparaison expérimental/numérique de la force appliquée par l'impactant pour des cellules de $500 \mathrm{~mm}$ remplies de granulats confinée (a) et non confinée (b) (Bertrand, 2006).

Experimental/simulation of the force applied by the boulder on $500 \mathrm{~mm}$ in height gravel cells in confined (a), and unconfined conditions (b) (Bertrand, 2006).

Les granulats sont modélisés par des agrégats insécables de sphères. Les agrégats sont initialement de forme parallélépipède rectangle et de distribution granulométrique identique aux granulats. Lors de leur mise en place dans la cellule, les agrégats sont positionnés de manière aléatoire et leurs arêtes sont coupées pour reproduire l'angularité des granulats. La dégradation lors du chargement de l'assemblée granulaire, principalement par fracturation, est prise en compte grâce à un modèle de contact interagrégats élastique parfaitement plastique. Ce modèle limite la valeur de force dans la direction normale au plan de contact. Les déformations plastiques sont localisées aux zones voisines des points de contact sans affecter le reste de l'agrégat. Au final, ce modèle permet de reproduire l'influence de la détérioration des granulats sur le comportement macroscopique de l'assemblée granulaire (Bertrand et al., 2005).

Le grillage est décrit par un ensemble de particules sphériques positionnées aux nœuds physiques de la maille, c'est à dire à l'intersection des brins. Les interactions physiques entre ces nœuds sont modélisées par le biais de forces d'interaction à distance, entre les partícules. Le modèle différencie les brins simples des brins torsadés, caractéristiques du grillage double torsion. Le comportement mécanique des brins est modélisé par une loi élastoplastique avec rupture, calée sur le comportement réel des brins. La réponse du modèle de grillage a été validée par comparaison avec des essais de traction sur bande large de grillage (Bertrand et al., 2006).

Une cage grillagée est obtenue par repliement de bandes de ce grillage, puis remplie avec les agrégats par application de la gravité, c'est-à-dire par pluviation. Cette cellule numérique est ensuite víbrée dans le but d'atteindre une porosité similaire à celle des essais expérimentaux.

Des essais de chargement pseudo-statique de cellules de $500 \mathrm{~mm}$ remplies de granulats confinées ont permis le calage du modèle, notamment le calage des paramètres relatifs aux contacts interagrégats. Le modèle a été validé à partir d'essais sur des cellules non confinées (Fig. 2). D'une cellule à l'autre les résultats montrent, comme en expérimental, une forte variabilité dont l'origine est la texture initiale de l'assemblage granulaire. La simulation numérique a par ailleurs montré la très forte influence de la forme des agrégats sur la réponse de la cellule (Bertrand et al., 2005).

A partir de ce modèle, calé, des simulations sous sollicitations dynamiques ont été possibles (Fig. 12). Les résultats relatifs à des impacts sur deux cellules de

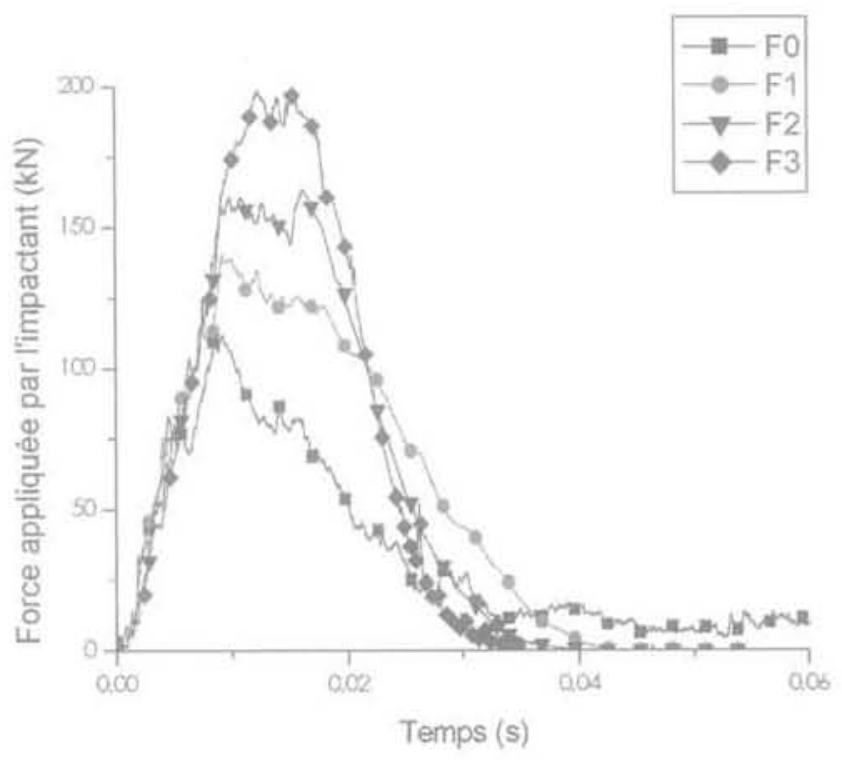

FIG.14 Modélisation de l'effet de confinement par application d'une force aux nœuds du grillage des faces latérales ; effet sur la réponse d'une cellule de $500 \mathrm{~mm}$ remplie de granulats impactée, pour différentes valeurs de force F0, F1, F2 et F3 valant respectivement $0,500,1000$ et $2000 \mathrm{~N}$. Modelling of the confining effect by applying a force on the node of the mesh: effect on the response during impact of a $500 \mathrm{~mm}$ in height gravel cell for different force values Fo, F1, F2 and $\mathrm{F} 3$ equalling $0,500,1,000$ and $2,000 \mathrm{~N}$ respectively. 
$500 \mathrm{~mm}$ remplies de granulats, confinées et non confinées, montrent une bonne concordance entre numérique et expérimental, aussi bien pour l'enveloppe globale de la courbe que pour les variations locales observées (Fig. 13).

Le modèle numérique de la cellule remplie de granulats apparait robuste. Il peut être utilisé pour simuler des situations difficiles à mettre en ceuvre expérimentalement. Un point important est l'influence de la présence de cellules contiguës sur la réponse de la cellule impactée. Ce point a pu être abordé numériquement en modélisant la force opposée par les cellules contiguës aux déformations latèrales de la cellule impactée. A chaque particule de grillage des faces latérales est appliquée une force normale au plan défini localement par celui-ci. En première approche les forces appliquées sont de valeur constante, estimée à partir des essais de chargement pseudo-statique sur cellules confinées : les simulations donnent un rapport de 0,13 entre la force de chargement et la force totale sur chaque face latérale. Connaissant les sollicitations sur les cellules lors des impacts et le nombre de nceuds par face, on déduit un ordre de grandeur de la force locale à appliquer sur chaque nœud. Des simulations d'impact ont été menées pour différentes valeurs de force (Fig. 14). En augmentant la force de confinement la durée de l'impact est réduite et la force maximale augmente, de façon analogue à ce qui est observé entre une cellule non confinée et une cellule confinée. Cette approche met clairement en évidence l'effet sur la réponse d'une cellule d'un confinement latéral non rigide, c'est-à-dire résultant de l'interaction avec d'autres cellules.

\section{Conclusion}

Pour comprendre le comportement sous impact de géocellules constitutives d'ouvrages à technique cellulaire, des essais ont été réalisés sur des cellules cubiques remplies de trois matériaux différents, en condition non confiné et confiné. L'influence du type de matériau de remplissage est mise en évidence notamment par l'interaction avec l'enveloppe de confinement, sollicitée en grandes déformations en l'absence de tirants. L'influence très importante des conditions aux limites a été mise en évidence. En l'absence de confinement, une cellule remplie de sable ou de mélange pneu-sable dissipe de façon plus uniforme l'énergie d'impact, notamment avec une plus grande sollicitation de l'enveloppe. Par contre, si la cellule est confinée, la possibilité de fracturation des granulats confère à ces matériaux un avantage par rapport aux matériaux fins. Le choix du matériau de remplissage des cellules pour obtenir une bonne atténuation des efforts dépend donc des conditions aux limites de la cellule, c'est à dire de l'interaction avec les cellules voisines au sein de l'ouvrage.

Ces essais ont par ailleurs permis de valider le modèle numérique et la modélisation à l'échelle de l'ouvrage peut alors être abordée, à partir d'un modèle constitutif de la cellule (Nicot et al., 2007).

Les résultats expérimentaux doivent être complétés en appliquant un confinement plus en accord avec le fonctionnement attendu dans un ouvrage. Sur la base de l'ensemble de ces résultats, il sera possible de définir les ouvrages expérimentaux de grandeur réelle à soumettre à impact, objectif du projet de recherche Rempare soutenu par l'ANR 2006.

\footnotetext{
REMERCIEMENTS

Globalement supportés par France Maccaferri SA, ces travaux sont réalisés en collaboration entre IUJJF-L3SR et le CemagrefETNA dans le cadre du GIS VOR-RNVO, qui a financé l'équipement du site utilisé pour ces expériences (Grés-sur-Aix, 73). La réalisation des essais présentés a été rendue possible gráce au soutien financier apporté par le Póle grenobloís des Risques naturels du conseil général de I'Tsère (CGI38-PGRN) et par la fourniture gracieuse des géotextiles par Bidim Geosynthetics SA. Les auteurs remercient vivement ces acteurs ainsi que foutes les personnes qui contribuent par leur intérèt à supporter et encourager cette recherche.
} 
Afnor-NF P 94-325-1 : Exécution des travaux géotechniques spéciaux - Ouvrages en gabion. Partie 1 : Ouvrages hors site aquatique. Afnor, 2004, 24 p.

Bathurst B.I., Karpurapu R. - Large-scale triaxial compression testing of geocell. reinforced granular soils. Geotechnica! testing journal, 1993, vol. 16, p. 296-303.

Bertrand D. Nicot F., Gotteland P. Lambert S.-Modelling a geo-composite cel. using discrete analysis. Computers and Geotechnics, 32. 2005, p. 564-577

Bertrand D. - Modélisation du comportement mécanique d'une structure cellulaire soumise à une sollicitation dvnamique localisée, Application aux structures de protection contre les éboulements rocheux. These de doctorat, université Joseph-Fourier Grenoble 1 Liriam, Cemagref. Grenoble, 2006, 197 p.

Bertrand D., Gotteland P., Lambert S., Nico F. Derache - Multi-scale modelling of cellular geo-composite structure under localized impact. Revue européenne de génie civil. (10)3, 2006, p. 309-322

Bertrand D. Gotteland P. Nicot F. - D.E.M. modelling of natural stones assembly confined in wire mesh. Powders \& Grains 2005, Rojo-Garcia Herrman and MoNamara Eds. Balkema. Stuttgart (Germany), vol. 1, 2005, p. 681-685.
Delhomme F, - Étude du comportement sous impact d'une structure pare-blocs en béton armé. Thèse de doctorat, université de Savoie, LOCIE: Chambéry. 2005, 234 p.

Desccudres F - Aspects géomécaniques des instabilités de falaises rocheuses et des chutes de blocs. Publications de la société suisse de mécanique des sols et des roches, 135, 1997, p. 3-11.

Gotteland P., Bertrand D., Lambert S. Nicot F. - Modelling an unusual geocomposite material barrier against a rockfall impact. Proc of IACMAG 2005, Torino (Italy), 2005a, p. 529-536.

Gotteland P.. Lambert S., Balachowski L. Strength characteristics of tyre chips. sand mixtures. Studia geotechnica et mechanica, vol. 27(1), 2005b, p. 55-66,

lizuka A. Kawai K. Kim E. R., Hirata M. Modeling of the confining effect due to the geosynthetic wrapping of compacted soil specimens. Geotextiles and geomembranes, vol. 22, 2004, p. 329-358. Lambert S., Gotteland P., Plé O., Bertrand D. Nicot F. - Modélisation du comportement mécanique de cellules de maté. riaux confinés. Journées nationales de Géotechnique et de Géologie. Lille (France), 2004, p. 219-226.

Montani Stoffel S - Sollicitation dynamique de la couverture des galeries de protection lors de chutes de blocs. Thèse de doctorat, EPFL, Lausanne, 1998, 180 p.

Nicot F., Gotteland P., Bertrand D., Lambert, S - Multi-scale approach to geocomposite cellular structures subjectec to rock impacts. International Journal for Numerical and Analytical Methods in Geomechanics, 2007 (à paraitre),

Oda M. Konishi J. Nemat-Nasser S. Experimental evaluation of strength of granular materials, effects of particle rolling. Mechanics of Materials, vol. 1. 1982, p. 269-283.

Pichler B., Hellmich C., Mang H.A. Impact of rocks onto gravel. Design and evaluation experiments, International Journal of Impact Engineering, vol. 31 . 2005, p. 559-578.

Radjai E., Wolf D.E., Jean M., Moreau J.J. Bimodal character of stress transmis. sion in granular packings. Physical Review Letters, Vol. 80(1)، 1998, p. 61-64.

Radiai F. Roux S.. Moreau J.J. - Contact forces in a granular packing. Chaos. vol. 9 (3). 1999, p. 544-550.

Tsoungui O., Vallet D., Charment J.C. Numerical model of crushing of grains inside two-dimensionnal aranular materials. Powder technology, vol. 105, 1999, p. 190-198. 